\title{
Salivary Interleukin-6 Levels among Polycystic Ovary Syndrome (Pcos) Patients with and Without Chronic Periodontitis - A Comparitive Study
}

\author{
Dr. Arun Varghese ${ }^{1}$, Dr. A. Julie Christy ${ }^{* 2}$, Dr. B. Pratheeba ${ }^{3}$, Dr. P. Saraswathy ${ }^{4}$, Dr. Sumathy Ravi ${ }^{5}$ \\ ${ }^{1}$ Sudha Hopsital, Opp Vadalur Sabai, Vadalur \\ ${ }^{2} \mathrm{PhD}$ Scholar, Bharath University (BIHER), Seliyur, Tambaram, Chennai \\ ${ }^{3}$ Professor, Department of Periodontology, Indra Gandhi Institute of Dental Sciences, Puducherry \\ ${ }^{4}$ Emeritus professor of anatomy, Tamil Nadu DR. M.G.R medical university, Guindy, Chennai \\ ${ }^{5}$ Professor and Head Department of Anatomy, Sri Balaji Dental College and Hospital, Chennai
}

Address for Correspondence: Dr. A. Julie Christy, BDS, MSc, (PhD); drjuliechristy @ gmail.com

Received 14 August 2019;

Accepted 04 September 2019;

Published 25 September 2019

\begin{abstract}
Background: Periodontitis is associates with various systemic disease one of which is PCOS. PCOS is a genetically complex endocrinopathy of uncertain etiology affecting women of reproductive age group which results in most common cause of anovulatory infertility, menstrual dysfunction and hirsutism. PCOS have close association with cardiometabolic risk profile, insulin resistance (IR), hyperinsulinemia, central obesity, dyslipidemia and increase prevalence of cardiovascular risk factors. The common pathway is the chronic low grade inflammation which is constituted by proinflammatory cytokine IL-6. Aim: To compare salivary IL-6 levels among patients with Polycystic ovary syndrome (PCOS) patients with chronic periodontitis and without Chronic Periodontitis. Materials and Methods: Newly diagnosed PCOS patients were selected for the study and the periodontal Parameters were recorded. Group A consist of 42 patients of PCOS with Periodontitis \& Group B consist of 42 patients of PCOS without Periodontitis. Salivary levels of IL-6 was compared between the two groups, and was assessed by Enzyme Linked Immuno Sorbent Assay (ELISA) kit (Bioassay). Results: BOP in group A was $1.40 \pm 0.40$ and in group B it was 0.91. \pm 0.18 ,PPD in group A was $4.23 \pm 0.134$ and in group B it was $1.30 \pm 0.06$, CAL in group A was $4.87 \pm 0.124$ and in group B was $1.30 \pm 0.06$, CAL in group A was $4.87 \pm 0.124$ and in group B was $1.30 \pm 0.06$. The values were statistically significant $(\mathrm{p}=<.001)$. IL-6 level in group A is $102.59 \pm 18.2$ and in group B it was $51.3 \pm 25.3$. Which implies there is an increase salivary IL-6 level in group A (PCOS with Periodontitis) than group B (PCOS without Periodontitis). Conclusion: Salivary IL-6 levels shows a double fold increase in Group A (PCOS with Periodontitis) than in group B ( PCOS without Periodontitis).
\end{abstract}

Keywords: salivary IL-6, PCOS, chronic periodontitis, insulin resistance, inflammatory cytokine

\section{Introduction}

Chronic periodontitis is a risk factor in the development of cardiovascular diseases, diabetes mellitus, occlusive respiratory diseases, rheumatoid arthritis and Poly Cystic ovary syndrome (PCOS) ${ }^{[1]}$ Dursun et al. reported for the first time an association between periodontal disease and PCOS. PCOS is the main cause of anovulatory infertility, and the most common gynecologic and endocrine condition among women in reproductive age. ${ }^{[2,3]}$

The pathogenomic state of chronic systemic inflammation and insulin resistance in both periodontitis and PCOS can be hypothesized as a converging channel associating the disorders. ${ }^{[4]}$ IR and hyperinsulinemia play a vital role in women with PCOS. ${ }^{[5]}$ IL-6 is an inflammatory mediator that stimulates osteoclast activity and bone resorption in periodontitis. ${ }^{[6]}$ Chronic periodontitis patients exhibit increased levels of pro inflammatory cytokines like TNF- $\alpha$, IL-1, IL-6. in serum and or in GCF. ${ }^{[7]}$ This chronic inflammatory phase results in IR. The levels of pro inflammatory cytokines can possibly be a marker or mediator of association between periodontitis and PCOS. ${ }^{[8]}$ Among the various cytokines implicated in pathology of periodontal inflammation and PCOS, IL- 6 , TNF- $\alpha$, hsCRP have gained greater attention as they are markers of systemic inflammation. ${ }^{[9]}$

Studies correlating levels of TNF- $\alpha$ and hsCRP with PCOS and periodontal status are present in literature. To the best of our knowledge there are no studies correlating IL-6 levels in PCOS and periodontitis patients. Our aim is to compare salivary IL-6 levels among Polycystic ovary syndrome (PCOS) patients with and without chronic periodontitis and our objective is to estimate the salivary IL-6 levels in participants with and without chronic 
periodontitis and to compare salivary IL-6 in both groups in newly diagnosed PCOS patients.

\section{Materials and Methods}

This study was conducted in Department of Periodontics, Indira Gandhi Institute of Dental Sciences, Puducherry. Department of Gynaecology, Mahatma Gandhi Medical College and research Institute, Pudhucherry. The study has been approved by the Institutional Ethics committee and Institutional Review Board (IGIDSIRB2015 NDP13PGATPAI).

Sample size is calculated by using $G$ Power software, in accordance to mean and standard deviation of the BOP, CAL, PPD of Porwal et al study is taken to determine the sample size and effect size was 0.73 . Based on this, sample size was calculated as 84 (42 in each group).

\section{Inclusion criteria}

Otherwise systemically healthy patients, newly diagnosed with PCOS according to Rotterdam criteria, Presence of $\geq 16$ natural teeth, PPD of $>3 \mathrm{~mm}, \mathrm{CAL}$ of $\geq 4 \mathrm{~mm}$, and if it is present more than $30 \%$ of the site is considered as chronic generalized periodontitis and less than $30 \%$ is considered as localized periodontitis, CAL 1-2 mm is mild, 3-4 is moderate, more than 5 is severe periodontitis, according to AAP classification (1999).

\section{Exclusion criteria}

Patient with history of thyroid dysfunction, Hyperprolactinemia, Androgen-secreting tumors to avoid misdiagnosis of PCOS, Patient with chronic inflammatory disease such as nephritic syndrome, chronic renal failure, significant cardiovascular disease, known type I/II diabetes mellitus, active cancer patients within past 5

years, Patient with adverse habits such as smoking, drinking, History of systemic antibiotic within 3 months, Periodontal treatment within 6 months and aggressive periodontitis. Patients who were categorized as obese and morbidly obese (according to WHO classification) were also excluded.

In this study the clinical parameter taken for periodontal status is BOP, PPD and CAL. Both the group individuals were examined by a Single examiner with UNC 15 probe and no.5 mouth mirror. BOP was recorded by modified sulcular bleeding index, Probing pocket depth was calculated from marginal gingiva to the base of the pocket, at six sites per tooth. Clinical Attachment level was calculated from CEJ to the base of the pocket, at six sites per tooth.

\section{Methodology}

Newly diagnosed subjects with PCOS were recruited for the study, the clinical periodontal examination was done and parameters recorded (BOP,PPD,CAL). Based on parameters grouped into group A (PCOS with periodontitis) and group B (PCOS without periodontitis), the unstimulated salivary samples were collected and preserved at at $-40^{\circ} \mathrm{C}$, the salivary samples were analysed by ELISA kit (BIOASSAY TECH) and results were analysed by independent $t$ test.

\section{Sample Collection}

Unstimulated saliva was collected in $5 \mathrm{ml}$ sterile container in PCOS patients and stored in $-40^{\circ} \mathrm{C}$, once the desired samples size was achieved, the samples are centrifuged at $3000 \mathrm{rpm}$ for 15 minutes. The supernatant saliva was collected in $1.5 \mathrm{ml}$ sterile vial through micropipette and stored at $-40^{\circ} \mathrm{C}$.

\section{Results}

\section{Table 1: Comparison of age, BOP, PPD, CAL and levels of IL-6 between Group A and Group B}

\begin{tabular}{|l|c|c|c|c|c|c|}
\hline \multirow{2}{*}{ Variable } & \multicolumn{2}{|c|}{ Group A $(\mathbf{n}=\mathbf{4 3})$} & \multicolumn{2}{c|}{ Group B (n=43) } & \multirow{2}{*}{ t-value } & \multirow{2}{*}{ p-value } \\
\cline { 2 - 6 } & Mean & SD & Mean & SD & & \\
\hline Age ( years) & 24.88 & 3.2 & 24.09 & 2.9 & 1.17 & 0.24 \\
\hline PPD $(\mathbf{m m})$ & 1.40 & 0.40 & 0.91 & 0.18 & 7.325 & $<.001$ \\
\hline CAL $(\mathbf{m m})$ & 4.23 & 0.134 & 1.30 & 0.06 & 164.6 & $<.001$ \\
\hline IL-6 $($ Pg/d) & 4.87 & 0.124 & 1.30 & 0.06 & 169.9 & $<.001$ \\
\hline Independent t-test used; $p$-value <.05 is significant; & 18.2 & 51.3 & 25.3 & 10.79 & $<.001$ \\
\hline
\end{tabular}

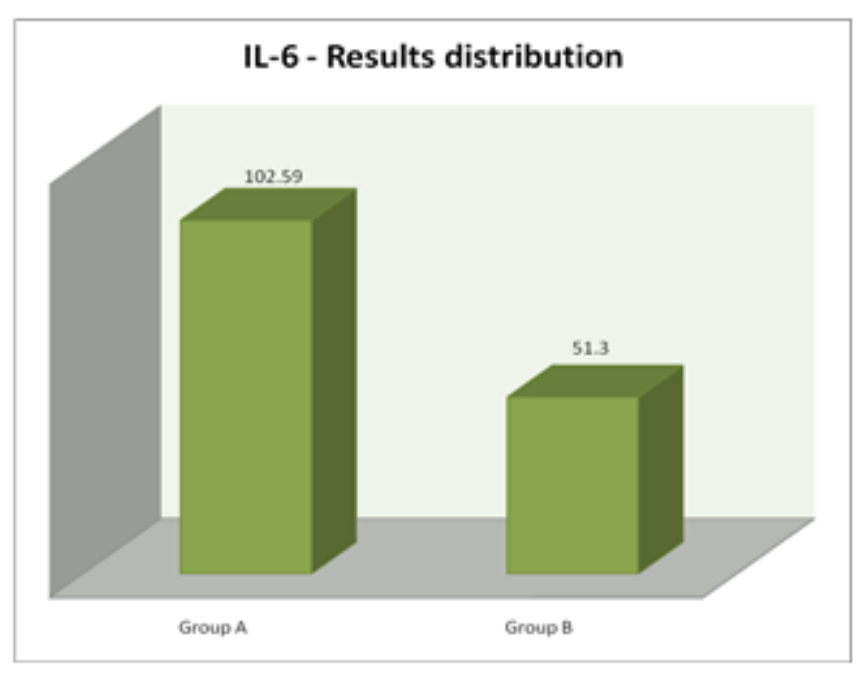

Graph 1 Il-6 Results Distribution

In our study, group A has mean age of $24.88 \pm 3.2$ years from reproductive age group between $18-30$ years and group B has a mean age of $24.09 \pm 2.9$ years. On comparing age between group A and group $B$ the values were not statistically significant. $(p=0.24)$.

Bleeding on probing calculated according to modified sulcular bleeding index and the mean BOP in group A was $1.40 \pm 0.40$ and in group B it was 0.91. \pm 0.18. On comparing BOP between group $\mathrm{A}$ and group $\mathrm{B}$, the values were statistically significant $(\mathrm{p}<.001)$.

The Probing Pocket Depth was measured with UNC 15 probe and marked to the nearest $0.5 \mathrm{~mm}$. the mean value in group A was $4.23 \pm 0.134$ and in group B it was $1.30 \pm 0.06$. On comparing PPD between group $\mathrm{A}$ and group $\mathrm{B}$, The values were statistically significant $(\mathrm{p}<.001)$. The mean value for $\mathrm{CAL}$ in group $\mathrm{A}$ was $4.87 \pm 0.124$ and in group $\mathrm{B}$ was $1.30 \pm 0.06$. On comparing CAL between group A and group B, The values were statistically significant $(\mathrm{p}<.001)$.

The mean IL-6 level in group A is $102.59 \pm 18.2$ and in group B it was $51.3 \pm 25.3$. On comparing IL-6 levels between group A and group $\mathrm{B}$, The values were statistically significant $(\mathrm{p}<.001)$, which implies there is an increase salivary IL-6 level in group A (PCOS with Periodontitis) compared to group B (PCOS without Periodontitis). 


\section{Discussion}

Periodontitis is chronic inflammatory condition which directly increases the production of proinflammatory cytokines such as TNF- $\alpha$, IL-6, IL-17, leads to the destruction of the periodontal tissues. ${ }^{[10,11]}$ The chronic nature of periodontal infection, inflammation and pathogenesis can cause effects elsewhere in the body, the most studied and linked diseases with periodontitis are diabetes mellitus, cardio vascular disease and adverse pregnancy outcomes. ${ }^{[12]}$ PCOS is considered to be a metabolic syndrome with cardiovascular, dyslipidemia, insulin-dependent diabetes, visceral obesity risk factors and endothelial dysfunction. ${ }^{[13,14,15]}$ Increase in Androgen production results in Insulin Resistance and Hyperinsulinemia. ${ }^{[16]}$ In PCOS patients increase production of proinflammatory cytokines such as TNF- $\alpha$, IL-6, IL-17 results in a state of constant low grade inflammatory response. This in turn can have adverse effects on the periodontal tissue. ${ }^{[17,18]}$

To the best of our knowledge only few studies have explored the levels of inflammatory mediators in PCOS Patients with and without periodontitis. ${ }^{[18,19]}$ There are no studies assessing the levels of salivary IL-6 in PCOS patients with periodontitis. In lieu with above, the present study assessed and compared the levels of IL-6 in PCOS patients with and without periodontitis. Frodge et al in 2008, taba M et al in 2005 stated that several inflammatory and immune mediators which are responsible for periodontal destruction can be detected in saliva. ${ }^{[20,21]}$ Pellegrini et al stated that quantifying biomarkers in saliva serve as a useful tool to predict an individual's susceptibility to periodontitis, to provide information for periodontal activity, and to monitor the effectiveness of periodontal therapy. ${ }^{[22]}$ Hence present study used saliva as a marker for IL6 levels.

The mean age group of the patient in group A and group B is $24.88 \pm 3.2$ and $24.09 \pm 2.9$ respectively which falls within the reproductive age for women. In the current study the mean BOP for Group A was $1.40 \pm 0.4$ and group B was $0.91 \pm 0.18$. PPD for group A was $4.23 \pm 0.134$ and group B was $1.30 \pm 0.06$. Cal for group A was $4.87 \pm 0.124$ and group B was $1.3 \pm 0.06$ all were statistically significant $(\mathrm{P}<0.001)$. Salivary IL-6 for group A was $102.59 \pm 18.2$ and group B was 51.3 \pm 25.3 . This reveals that IL-6 levels were two fold increased in PCOS patients with periodontitis than in PCOS patients without periodontitis.

Noh MK et al in 2013 showed increased IL-6 level in periodontitis patients. ${ }^{[23]}$ Gabriela et al in 2014 stated that there is association between IL- 6 and periodontitis and IL- 6 is an important biomarker for periodontitis. ${ }^{[24]}$ Costa AM et al in 2010 suggested that there is an increase in Salivary IL-6 level in periodontitis patient with diabetes than periodonitits without diabetes. ${ }^{[25]}$ Tarkun I et al in 2006 in a case control study to evaluate the levels of IL-6 in PCOS patients and found that PCOS group had elevated IL-6 levels than the controls. ${ }^{[26]}$ Kucuk M et al in 2014, compared IL-6 levels in patients with and without PCOS and found increased levels of IL-6 in PCOS group. ${ }^{[27]}$ Peng Z et al in 2016, in a meta analysis found that IL- 6 levels were increased in PCOS group as compared with control group. ${ }^{[28]}$

Our study reveals that IL-6 levels increased with increase in periodontal inflammation in patients with PCOS. IL-6 is one of the very important cytokines associated with insulin resistance. ${ }^{[29]}$ IL-6 induces insulin resistance by decreased tyrosine phosphorylation of IR substrate (IRS)-1 and decreased association of the p85 subunit of phosphatidylinositol 3-kinase with IRS-1 in response to physiologic insulin levels. In addition, insulin-dependent activation of Akt, important in mediating insulin's downstream metabolic actions, is markedly inhibited by IL-6 treatment. ${ }^{[29]}$ This results in insulin resistance of insulin receptors to insulin action in liver. However few reports suggest a beneficial role of IL-6 in skeletal muscle. ${ }^{[30,31]}$

Furthermore, the effects of the cytokine are seemingly influenced by whether it is present acutely or chronically; the latter is the setting associated with insulin resistance. ${ }^{[32]}$ This results in high insulin levels in circulation as compensation leading to hyperinsulinemia. Since periodontitis is a chronic inflammatory condition this leads to chronic increased level s of IL-6 that might increase insulin resistance. This study reflects the importance of periodontal health and prevention of periodontal disease to avoid increase in insulin resistance in PCOS patients. Hyperinsulinaemia promotes hyperandrogenism in PCOS by two distinct and independent mechanisms: (i) by increasing circulating ovarian androgens; and (ii) by directly reducing serum sex hormonebinding globulin concentrations. ${ }^{[33]}$ The net result of these actions is to increase circulating free testosterone concentrations.

Limited evidence suggests that hyperinsulinaemia might also promote ovarian androgen production by influencing pituitary release of gonadotrophins. ${ }^{[34,35,36]}$ This hyperandrogenism has direct effects on development, progression \& treatment resistance in PCOS patients. ${ }^{[37]}$ Treatment aimed in bringing down hyperinsulinemia, such as use of drugs like metformin resulted in decreased levels of IL- 6 post treatment. ${ }^{[38]}$ This also resulted in decreased levels of circulating androgens. The clinical implication of these findings is that amelioration of hyperandrogenism in women with PCOS may be achieved by interventions which improve insulin sensitivity and reduce circulating insulin. Such measures might include, but are not limited to, weight loss, dietary modification, and insulin-sensitizing medications. ${ }^{[39]}$

Vidal $\mathrm{F}$ et al in 2009 reported that non-surgical periodontal therapy was effective in improving periodontal clinical data and in reducing the plasma levels of IL-6 in patients with severe periodontitis. ${ }^{[40]}$ Mammen $\mathbf{J}$ et al in 2017 described that effective periodontal therapy reduced insulin resistance and improved periodontal health status and insulin sensitivity in patients with type II DM and chronic periodontitis. ${ }^{[41]}$ This proves that periodontal therapy is an important means to decrease levels of IL6 and improve insulin sensitivity in such patients. Hence comprehensive periodontal screening and therapy is very essential in patients with PCOS to improve insulin sensitivity and thereby decreasing hyperandrogenism.

The limitations in our study are that Chronic periodontitis without PCOS was not included and lack of interventional periodontal therapy to check for reduction in levels of IL-6 which might reflect in reduced Insulin resistance. Further studies with a larger sample size is required to confirm the association between IL-6 levels in PCOS patients with periodontitis.

\section{Conclusion}

Results show that there is an increase in the salivary IL-6 levels in PCOS patient with periodontitis by two fold as compared to those without periodontitis. This study reflects the importance of periodontal health and prevention of periodontal disease so as to minimize insulin resistance in PCOS patients with periodontitis. Periodontal therapy is an important means to decrease levels of IL6 and improve insulin sensitivity in such patients. Hence comprehensive periodontal screening and therapy is essential in patients with PCOS to improve insulin sensitivity, thereby decreasing hyperandrogenism.

\section{Disclaimers}


No Disclaimers

\section{References}

1. Legro RS. Polycystic ovary syndrome and cardiovascular disease: a premature association?. Endocrine reviews. 2003;24:302-12.

2. Dursun E, Akalın FA, Güncü GN, Çınar N, Aksoy DY, Tözüm TF, Kılınc K, Yıldız BO. Periodontal disease in polycystic ovary syndrome. Fertility and sterility. 2011;95:320-3.

3. Uitto VJ, Overall CM, McCulloch C. Proteolytic host cell enzymes in gingival crevice fluid. Periodontology 2000. 2003;31:77-104.

4. Porwal S, Tewari S, Sharma RK, Singhal SR, Narula SC. Periodontal status and high-sensitivity C-reactive protein levels in polycystic ovary syndrome with and without medical treatment. Journal of periodontology. 2014;85:1380-9.

5. Baillargeon JP, Iuorno MJ, Nestler JE. Insulin sensitizers for polycystic ovary syndrome. Clinical obstetrics and gynecology. 2003;46:325-40.

6. Hienz SA, Paliwal S, Ivanovski S. Mechanisms of bone resorption in periodontitis. Journal of immunology research.2015.

7. Shen YX, Guo SJ, Wu YF. Oxidative stress and antioxitant therapy of chronic periodontitis. Chinese journal of stomatology. 2016;51:442-6.

8. Cekici A, Kantarci A, Hasturk H, Van Dyke TE. Inflammatory and immune pathways in the pathogenesis of periodontal disease. Periodontology 2000. 2014;64:5780.

9. Kellesarian SV, Malignaggi VR, Kellesarian TV, AlKheraif AA, Alwageet MM, Malmstrom H, Romanos GE, Javed F. Association between periodontal disease and polycystic ovary syndrome: a systematic review. International journal of impotence research. 2017;29:8995.

10. Williams RC, Offenbacher S. Periodontal medicine: the emergence of a new branch of periodontology. Periodontology 2000. 2000;23:9-12.

11. Hasturk H, Kantarci A, Van Dyke TE. Oral inflammatory diseases and systemic inflammation: role of the macrophage. Frontiers in immunology. 2012;3.

12. Kampits C, Montenegro MM, Ribeiro IW, Furtado MV, Polanczyk CA, RÖSING CK, HAAS A. Periodontal disease and inflammatory blood cytokines in patients with stable coronary artery disease. Journal of Applied Oral Science. 2016;24:352-8.

13. Ebejer K, Calleja-Agius J. The role of cytokines in polycystic ovarian syndrome. Gynecological Endocrinology. 2013;29:536-40.

14. Ovalle F, Azziz R. Insulin resistance, polycystic ovary syndrome, and type 2 diabetes mellitus. Fertility and sterility. 2002;77:1095-105.

15. Wild RA, Carmina E, Diamanti-Kandarakis E, Dokras A, Escobar-Morreale HF, Futterweit W, Lobo R, Norman RJ, Talbott E, Dumesic DA. Assessment of cardiovascular risk and prevention of cardiovascular disease in women with the polycystic ovary syndrome: a consensus statement by the Androgen Excess and Polycystic Ovary Syndrome (AE-PCOS) Society. The
Journal of Clinical Endocrinology \& Metabolism. 2010;95:2038-49.

16. Samy N, Hashim M, Sayed M, Said M. Clinical significance of inflammatory markers in polycystic ovary syndrome: their relationship to insulin resistance and body mass index. Disease markers. 2009;26:163-70.

17. Kelly CC, Lyall H, Petrie JR, Gould GW, Connell JM, Sattar N. Low grade chronic inflammation in women with polycystic ovarian syndrome. The Journal of Clinical Endocrinology \& Metabolism. 2001;86:2453-5.

18. Kalyan S, Patel MS, Kingwell E, Côté HC, Liu D, Prior JC. Competing Factors Link to Bone Health in Polycystic Ovary Syndrome: Chronic Low-Grade Inflammation Takes a Toll. Scientific Reports. 2017;7.

19. Kellesarian SV, Malignaggi VR, Kellesarian TV, AlKheraif AA, Alwageet MM, Malmstrom H, Romanos GE, Javed F. Association between periodontal disease and polycystic ovary syndrome: a systematic review. International journal of impotence research. 2017;29:8995.

20. Frodge BD, Ebersole JL, Kryscio RJ, Thomas MV, Miller CS. Bone remodeling biomarkers of periodontal disease in saliva. Journal of periodontology. 2008;79:1913-9.

21. Taba M, Jin Q, Sugai JV, Giannobile WV. Current concepts in periodontal bioengineering. Orthodontics \& craniofacial research. 2005;8:292-302.

22. Pellegrini GG, Chaves MM, Fajardo MA, Ponce GM, Toyos GI, Lifshitz F, Friedman SM, Zeni SN. Salivary bone turnover markers in healthy pre-and postmenopausal women: daily and seasonal rhythm. Clinical oral investigations. 2012 Apr 1;16(2):651-7.

23. Noh MK, Jung M, Kim SH, Lee SR, Park KH, Kim DH, Kim HH, Park YG. Assessment of IL 6, IL 8 and TNF $\alpha$ levels in the gingival tissue of patients with periodontitis. Experimental and therapeutic medicine. 2013;6:847-51.

24. Gabriela Teixeira F, Mendonça SA, Menezes Oliveira K, Barbosa dos Santos D, Miranda Marques L, Mendonça Amorim M, de Souza Gestinari R. Interleukin-6 c.174G> C polymorphism and periodontitis in a Brazilian population. Molecular biology international. 2014.

25. Costa AM, Guimarães MC, De Souza ER, Nóbrega OT, Bezerra AC. Interleukin-6 (G-174C) and tumour necrosis factor-alpha (G-308A) gene polymorphisms in geriatric patients with chronic periodontitis. Gerodontology. 2010;27:70-5.

26. Tarkun İ, Çetinarslan B, Türemen E, Cantürk Z, Biyikli M. Association between circulating tumor necrosis factor-alpha, interleukin-6, and insulin resistance in normal-weight women with polycystic ovary syndrome. Metabolic syndrome and related disorders. 2006;4:122-8.

27. Küçük M, Altınkaya SÖ, Nergiz S, Sezer SD, Yüksel H, Bağlı İ, Yıldız G. Interleukin-6 levels in relation with hormonal and metabolic profile in patients with polycystic ovary syndrome. Gynecological Endocrinology. 2014;30:423-7.

28. Peng Z, Sun Y, Lv X, Zhang H, Liu C, Dai S. Interleukin-6 Levels in Women with Polycystic Ovary Syndrome: A Systematic Review and Meta-Analysis. PloS one. 2016;11:0148531.

29. Senn JJ, Klover PJ, Nowak IA, Mooney RA. Interleukin6 induces cellular insulin resistance in hepatocytes. Diabetes. 2002;51:3391-9. 
30. Muñoz-Cánoves P, Scheele C, Pedersen BK, Serrano AL. Interleukin-6 myokine signaling in skeletal muscle: a double-edged sword? The FEBS journal. 2013;280:4131-48.

31. Belizário JE, Fontes-Oliveira CC, Borges JP, Kashiabara JA, Vannier E. Skeletal muscle wasting and renewal: a pivotal role of myokine IL-6. SpringerPlus. 2016;5:619.

32. Kim JH, Bachmann RA, Chen J. Interleukin-6 and insulin resistance. Vitamins \& Hormones. 2009;80:61333.

33. Nestler JE. Insulin regulation of human ovarian androgens. Human Reproduction. 1997;12:53-62.

34. Buggs C, Rosenfield RL. Polycystic ovary syndrome in adolescence. Endocrinology and metabolism clinics of North America. 2005;34:677.

35. Blank SK, Helm KD, McCartney CR, Marshall JC. Polycystic ovary syndrome in adolescence. Annals of the New York Academy of Sciences. 2008;1135:76-84.

36. Franks S. Polycystic ovary syndrome in adolescents. International Journal of obesity. 2008;32.

37. Rojas J, Chávez M, Olivar L, Rojas M, Morillo J, Mejías J, Calvo M, Bermúdez V. Polycystic ovary syndrome, insulin resistance, and obesity: navigating the pathophysiologic labyrinth. International Journal of reproductive medicine. 2014.

38. Luque-Ramirez M, Escobar-Morreale HF. Treatment of polycystic ovary syndrome (PCOS) with metformin ameliorates insulin resistance in parallel with the decrease of serum interleukin- 6 concentrations. Hormone and metabolic research. 2010;42:815-20.

39. Nestler JE. Insulin regulation of human ovarian androgens. Human Reproduction. 1997;12:53-62.

40. Vidal F, Figueredo CM, Cordovil I, Fischer RG. Periodontal therapy reduces plasma levels of interleukin6, C-reactive protein, and fibrinogen in patients with severe periodontitis and refractory arterial hypertension. Journal of periodontology. 2009;80:786-91.

41. Mammen J, Vadakkekuttical RJ, George JM, Kaziyarakath JA, Radhakrishnan C. Effect of nonsurgical periodontal therapy on insulin resistance in patients with type Ii diabetes mellitus and chronic periodontitis, as assessed by C-peptide and the Homeostasis Assessment Index. Journal of investigative and clinical dentistry. 2017;8. 